\title{
Premature Epiphyseal Closure of the Lower Extremities Contributing to Short Stature after cis-Retinoic Acid Therapy in Medulloblastoma: A Case Report
}

\author{
Jessica J. Noyes ${ }^{a}$ Michael A. Levine $^{b}$ Jean B. Belasco ${ }^{a}$ Sogol Mostoufi-Moaba, b \\ ${ }^{a}$ Department of Pediatrics, Division of Oncology, and b ${ }^{b}$ Department of Pediatrics, Division of Endocrinology, The \\ Children's Hospital of Philadelphia, The University of Pennsylvania Perelman School of Medicine, Philadelphia, Pa., USA
}

\section{Established Facts}

- Prolonged cis-retinoic acid (RA) exposure results in premature epiphyseal closure of the lower extremities with compromised growth in pediatric patients.

\section{Novel Insights}

- Pediatric medulloblastoma survivors treated with cis-RA are at significant risk of growth failure and compromised final adult height due to concurrent radiation-induced growth hormone deficiency, radiation damage to the spinal bones, and cis-RA-associated premature closure of the lower-extremity growth plates.

\section{Key Words}

Hyena disease $\cdot$ cis-Retinoic acid $\cdot$ Radiation-induced growth hormone deficiency · Late effects · Epiphyseal closure

\begin{abstract}
Background: Prolonged cis-retinoic acid (RA) exposure contributes to premature epiphyseal closure. cis-RA is administered in various treatment regimens for pediatric cancers, thus increasing the risk for bone deformities and compromised growth. Results: We present a case of premature epiphyseal closure in a 9-year-old female with a history of medulloblastoma and treatment with a multimodal regimen including cis-RA. She was subsequently diagnosed with radiation-induced endocrine late effects including hypothyroid-
\end{abstract}

ism and growth hormone deficiency (GHD). Seven months after initiation of GH therapy, an increased prominence of the wrists and knees combined with a deceleration in growth velocity prompted further evaluation; radiographs revealed bilateral premature closure of the distal femur and proximal tibia growth plates despite normal left wrist bone age. Conclusion: High doses of vitamin A and its analogs are linked to premature closure of the lower-extremity growth plates in animals and children. Pediatric brain tumor patients are at increased risk of growth failure due to concurrent radiation-induced GHD, damage to the spinal bones, and cisRA-associated premature closure of the lower-extremity growth plates, with significant reduction in adult stature. A better appreciation of the detrimental effect of cis-RA on the growing skeleton is needed to monitor at-risk patients and to provide timely interventions.

(c) 2015 S. Karger AG, Basel

\section{KARGER}

E-Mail karger@karger.com

www.karger.com/hrp
(C) 2015 S. Karger AG, Basel

1663-2818/15/0851-0069\$39.50/0
Sogol Mostoufi-Moab, MD, MSCE

The Children's Hospital of Philadelphia

3535 Market Street

Philadelphia, PA 19104 (USA)

E-Mail moab@email.chop.edu 


\section{Introduction}

Vitamin A toxicity was noted in the 1940s, when parents accidentally overdosed their children with large quantities of newly accessible vitamins. Caffey [1] first described skeletal deformities including swollen limbs, pain, and radiographic changes from periostitis in children following administration of excess vitamin A. Subsequently, Pease [2] reported seven infants with premature epiphyseal closure due to chronic vitamin A overexposure, with irreversible growth arrest of the long bones.

In the 1990s, an unfamiliar disease in calves was identified as 'hyena disease', named for the calves' appearance and gait resembling those of a hyena. Woodard et al. [3] discovered excess vitamin $\mathrm{A}$ in grain as a contributing cause and demonstrated that vitamin $\mathrm{A}$ at higher doses led to growth plate closure that preferentially affected the lower limbs of the calves, thereby making them resemble a hyena, where the front legs are longer than the hind ones, giving the back a sloping appearance.

cis-Retinoic acid (RA), a vitamin A derivative, is administered for the treatment of selective pediatric cancers, with occasional reports of adverse skeletal effects including advanced bone age and premature closure of the growth plates [4-6]. These defects manifest as an acquired chondrodysplasia in experimental animal models where animals are given high doses of vitamin $\mathrm{A}$, with growth plate thinning, premature ossification of column carti- lage, and markedly reduced longitudinal bone growth even before premature growth plate closure [3, 7-9], likely through wnt/catenin pathway activation or antagonizing fibroblast growth factor receptor signaling.

A new treatment regimen for medulloblastoma includes cis-RA to induce cellular apoptosis $[10,11]$. The late effects of cis-RA in medulloblastoma patients have not been extensively studied. By contrast, the deleterious effects of craniospinal radiation on growth, through both growth hormone deficiency (GHD) and damage to spinal bone cells, are well recognized. We report a case of 'hyena disease' in a medulloblastoma survivor with prolonged cis-RA exposure and craniospinal radiation to highlight the multifactorial basis for growth failure. Parental consent and patient assent were obtained for this case report.

\section{Case Report}

The patient, a 9-year-old Caucasian female, was first diagnosed with medulloblastoma at age 3 years. Treatment included surgical resection of the tumor followed by systemic chemotherapy (vincristine, lomustine, cisplatin, carboplatin, cyclophosphamide, and etoposide) and craniospinal radiation (2,340 cGy) with boost to the posterior fossa (total dose 5,900 cGy). cis-RA was subsequently administered for eleven 14 -day cycles $\left(180 \mathrm{mg} / \mathrm{m}^{2}\right.$ per day) over a 13-month period for a cumulative dose of $17,360 \mathrm{mg}(24,640 \mathrm{mg} /$ $\mathrm{m}^{2}$ ). She completed medulloblastoma therapy at age 5.6 years.

At age 6 years, she was diagnosed with radiation-induced central hypothyroidism and GHD. She was treated with levothyroxine
Fig. 1. This graph shows the patient's time of diagnosis (Dx) and surgery (Sx), along with duration of radiation therapy (RT), chemotherapy (Chemo Tx), and GH treatment (GH Tx). The patient's growth chart reveals a height trajectory far below the 5 th percentile (mid-parental height of 65 inches) with limited height increase before the initiation of $\mathrm{GH}$ at approximately age 7 years. Height velocity briefly increased with the initiation of GH therapy but faltered shortly afterwards, despite continued $\mathrm{GH}$ treatment, due to prematurely closed epiphyseal growth plates of the lower extremities.

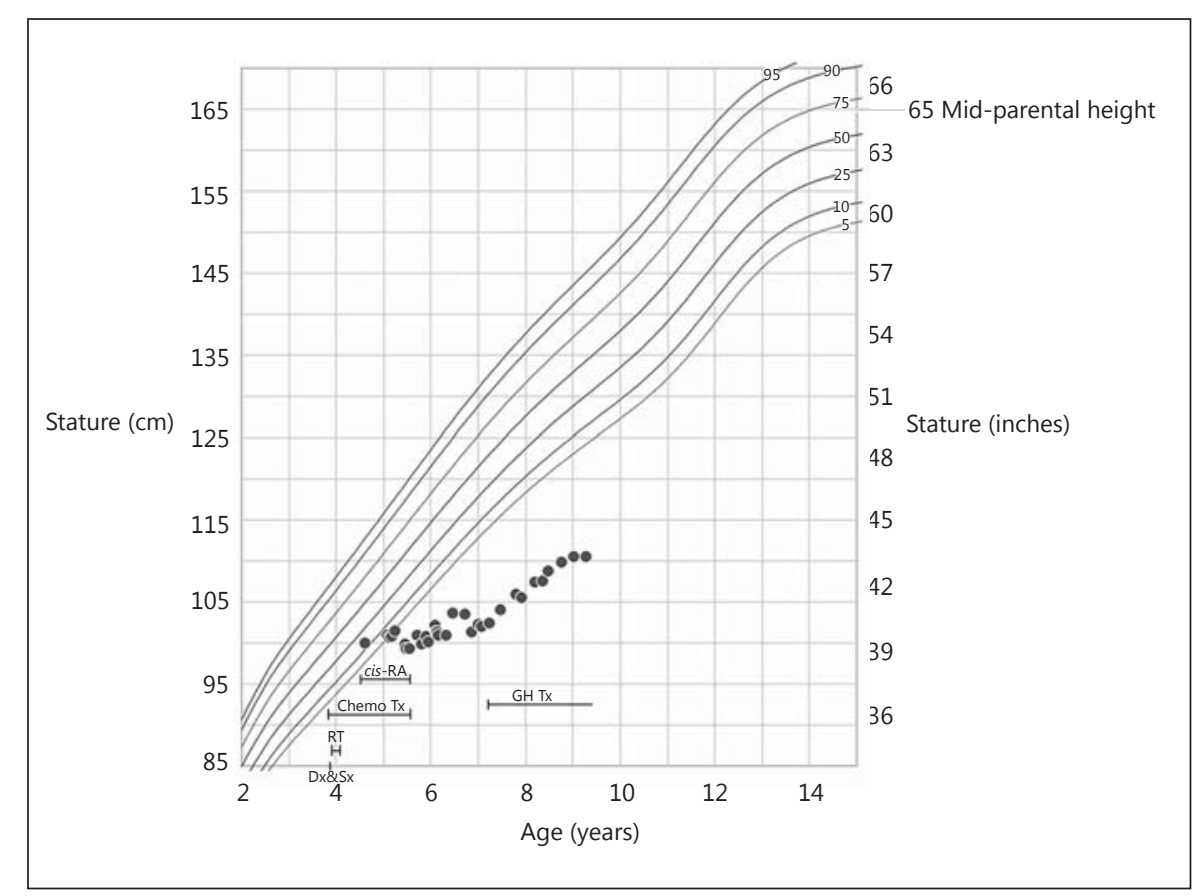


with subsequent biochemical euthyroidism. Given tumor remission status and an abnormal height velocity of $0.94 \mathrm{~cm} /$ year, $\mathrm{GH}$ therapy was initiated at age 7 years $(0.3 \mathrm{mg} / \mathrm{kg} /$ week $)$ with a subsequent increased height velocity of $3.6 \mathrm{~cm}$ growth $/ 7$ months; however, 7 months after initiation of GH therapy, increased bony prominence was noted at the wrists and knees, concomitant with declining height velocity (1.6 cm growth/5 months). Physical examination and radiographs were significant for leg length discrepancy: the right lower extremity measured $1.75 \mathrm{~cm}$ shorter than the left (right femur $24.9 \mathrm{~cm}$, right tibia $19.6 \mathrm{~cm}$, right lower extremity $44.5 \mathrm{~cm}$, left femur $25.25 \mathrm{~cm}$, left tibia $21 \mathrm{~cm}$, and left lower extremity $46.25 \mathrm{~cm})$. Prior to initiation of GH therapy, height $(102 \mathrm{~cm}$; fig. 1) and arm span $(102 \mathrm{~cm})$ were similar, with an upper-segment/lower-segment ratio of 1; 7 months after initiation of GH therapy, height increased minimally $(105.6 \mathrm{~cm})$ compared to the more significant increase in arm span $(109.2 \mathrm{~cm})$, with the upper- segment/lower-segment ratio remaining at 1 . Radiographs revealed poorly visualized growth plates in both distal and proximal femurs and tibias (fig. 2a, b), raising concern for premature closure of the physes. This lower-limb growth plate closure would normally be observed at the time of late-stage puberty. By contrast, all bone ages using left hand and wrist radiographs over the preceding 2 years displayed open growth plates (fig. 2c, d).

\section{Discussion}

13-cis-RA is a highly effective anticancer agent that induces cytodifferentiation and apoptosis as well as inhibition of angiogenesis and oncogene expression [12].
Fig. 2. Radiographs of the knee (a) and ankles (b) at age 8.4 years revealed metaphyseal widening with diffuse skeletal demineralization and poor visualization of the growth plates at the left and right distal and proximal femurs and tibias, demonstrating premature closure of the epiphyseal growth plates. In contrast to closed lower-extremity growth plates, bone ages from January 1, 2013 (chronological age 7 years; c), and April 24, 2014 (chronological age 8.4 years; d), were normal and less than 2 standard deviations below chronological age with open growth plates.
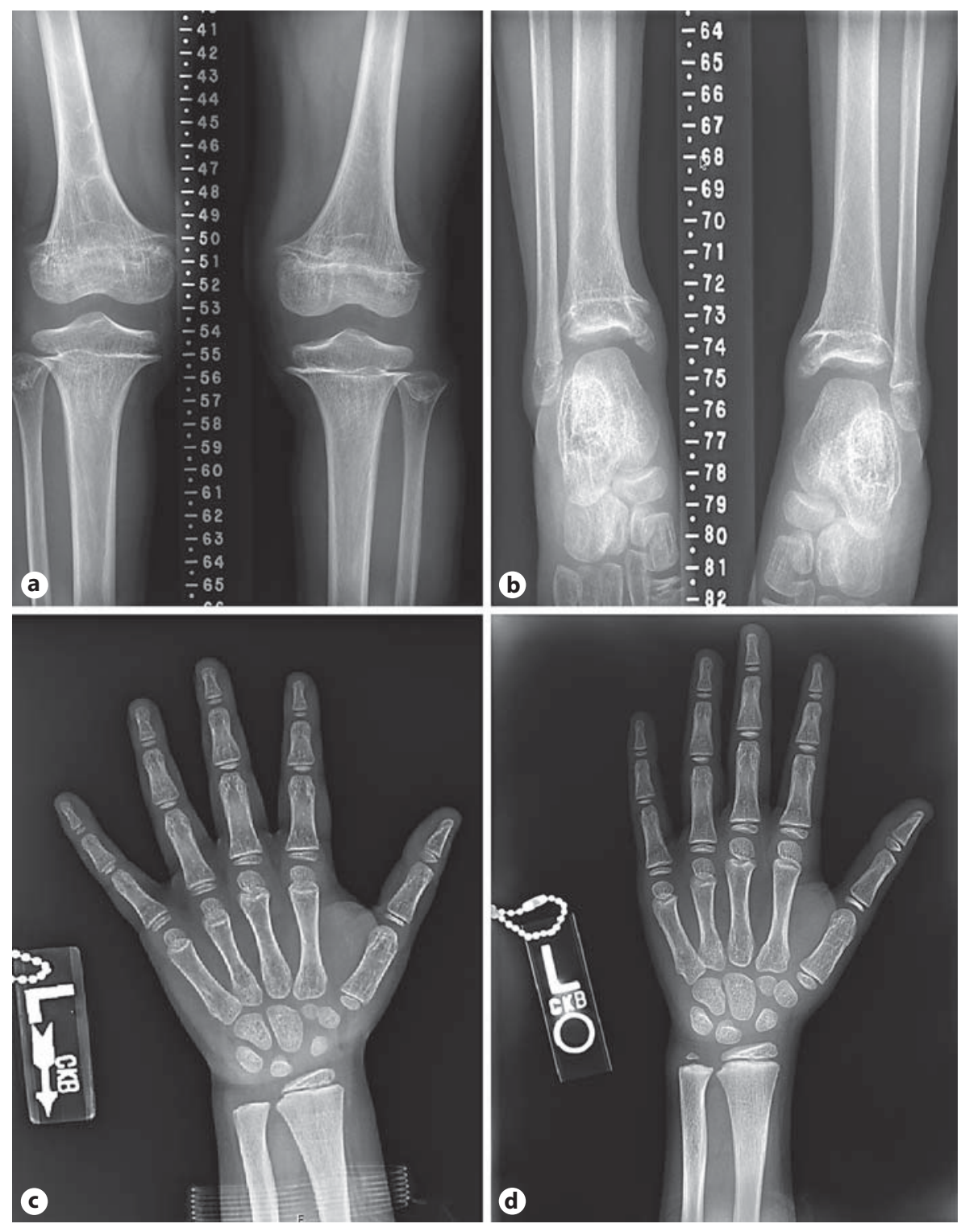
13-cis-RA is used in advanced therapies for neuroblastoma, acute promyelocytic leukemia [13], and high-risk medulloblastoma [11].

Despite the significant positive impact of 13-cis-RA on survival, acute and chronic toxicities are reported: hypervitaminosis A (characterized by weight loss, fever, alopecia, bone and joint pain, hyperostosis, thrombocytopenia, and elevated cerebral fluid pressure) [12] and premature epiphyseal closure [7]. Premature epiphyseal closure is a labeled side effect of 13-cis-RA, related to dose, age at exposure, and treatment duration with more pronounced effects in young individuals exposed to higher doses [7]. While most untoward effects are reversible after treatment discontinuation [7, 12], 13-cis-RA can have permanent deleterious effects on the developing skeleton [7].

Nevertheless, to date, few case reports have described adverse skeletal consequences of 13-cis-RA in children treated for neuroblastoma, including one which described declining growth velocity and epiphyseal closure $[5,6]$. In a retrospective analysis of 32 neuroblastoma survivors, Hobbie et al. [4] reported advanced bone age or prematurely closed epiphyses on left hand/wrist radiographs in $29 \%$ of patients treated with 13-cis-RA compared to the group without cis-RA exposure.

In animal models, increased doses of vitamin A result in skeletal defects. In rats, vitamin A toxicity causes calcification of the epiphyseal resting zone, with subsequent complete disappearance of the growth plate $[7,9]$. In calves, vitamin A toxicity results in 'hyena disease', where premature epiphyseal closure of the hind limbs and the ensuing abnormal growth result in a gait disturbance resembling hyenas $[3,8]$. Treated calves exhibit elevated levels of 13-cis-RA, a RA derivative [3].

Our patient experienced prolonged 13-cis-RA exposure as part of medulloblastoma therapy and demonstrated growth failure despite treatment with GH and premature epiphyseal growth plate closure that preferentially affected the lower extremities. To our knowledge, no prior reports in the medical literature ascertain the longterm skeletal side effects of high-dose cis-RA exposure in pediatric medulloblastoma patients.

Growth failure is a well-known side effect of craniospinal radiation, due in part to GHD [14]. While children with isolated GHD demonstrate proportionate short stature, the growth pattern following craniospinal radiation is notable for compromised growth in the upper segment compared to the lower segment from radiation damage to spinal bone cells. On the other hand, cis-RA disproportionately impacts lower-segment growth. Our patient had a proportionate short stature at the time of GH initiation and showed only a modest height increment, likely due to limited growth of the upper segment from irradiationinduced toxicity to spinal bone cells, and premature epiphyseal closure of the lower-extremity growth plates as a consequence of prior treatment with 13-cis-RA. The remarkable increase in arm span indicates a robust response to $\mathrm{GH}$ in the upper extremities, which are relatively spared from 13-cis-RA treatment, as indicated by age-appropriate bone ages of the left wrist (fig. 2c, d). Therefore, proportionate measures of both upper and lower segments, as well as arm span, should be monitored closely in children with growth failure following cancer treatment.

This case demonstrates premature lower-extremity epiphyseal growth plate closure with normal bone age in the wrist, indicating that conventional methods of determining bone age, i.e. left hand/wrist radiograph, are inadequate when evaluating growth in patients treated with high-dose 13-cis-RA, particularly those who are receiving $\mathrm{GH}$. With future inclusion of 13-cis-RA in different pediatric cancer treatment regimens, a better understanding of its detrimental effects on the pediatric skeleton is needed to identify at-risk subpopulations for timely interventions. Therefore, providers caring for childhood cancer patients treated with 13-cis-RA should be aware of its potential adverse impact on the developing skeleton and must take care to evaluate growth and epiphyseal status in a comprehensive manner.

\section{Acknowledgements}

This study was supported by NIH grant K07 CA166177 (S.M.-M.).

\section{Disclosure Statement}

All authors have no financial relationships relevant to this article and no potential conflicts of interest to disclose.

References

1 Caffey J: Chronic poisoning due to excess of vitamin $\mathrm{A}$; description of the clinical and roentgen manifestations in seven infants and young children. Am J Roentgenol Radium Ther 1951;65:12-26.

2 Pease $\mathrm{CN}$ : Focal retardation and arrestment of growth of bones due to vitamin A intoxication. JAMA 1962;182:980-985.

3 Woodard JC, Donovan AG, Eckhoff C: Vitamin (A and D)-induced premature physeal closure (hyena disease) in calves. J Comp Pathol 1997;116:353-366.
72 
4 Hobbie WL, Mostoufi SM, Carlson CA, Gruccio D, Ginsberg JP: Prevalence of advanced bone age in a cohort of patients who received cis-retinoic acid for high-risk neuroblastoma. Pediatr Blood Cancer 2011;56:474-476.

5 Inamo Y, Suzuki T, Mugishima H: A case of growth failure caused by 13-cis-retinoic acid administration after bone marrow transplantation for neuroblastoma. Endocr J 1999; 46(suppl):S113-S115.

-6 Nishimura G, Mugishima H, Hirao J, Yamato M: Generalized metaphyseal modification with cone-shaped epiphyses following longterm administration of 13-cis-retinoic acid. Eur J Pediatr 1997;156:432-435.

7 DiGiovanna JJ: Isotretinoin effects on bone. J Am Acad Dermatol 2001;45:S176-S182.

$\checkmark 8$ Rothenberg AB, Berdon WE, Woodard JC, Cowles RA: Hypervitaminosis A-induced premature closure of epiphyses (physeal obliteration) in humans and calves (hyena disease): a historical review of the human and veterinary literature. Pediatr Radiol 2007;37: 1264-1267.

-9 Kodaka T, Takaki H, Soeta S, Mori R, Naito Y: Local disappearance of epiphyseal growth plates in rats with hypervitaminosis A. J Vet Med Sci 1998;60:815-821.

10 Hallahan AR, Pritchard JI, Chandraratna RA, Ellenbogen RG, Geyer JR, Overland RP, Strand AD, Tapscott SJ, Olson JM: BMP-2 mediates retinoid-induced apoptosis in medulloblastoma cells through a paracrine effect. Nat Med 2003;9:1033-1038.

11 Olson J; Children's Oncology Group: Chemotherapy and radiation therapy in treating young patients with newly diagnosed, previously untreated, high-risk medulloblastoma. http://www.cancer.gov/clinicaltrials/search/ view ?cdrid $=511991 \&$ version $=$ HealthProfessi onal (accessed December 16, 2014).

12 Miller WH Jr: The emerging role of retinoids and retinoic acid metabolism blocking agents in the treatment of cancer. Cancer 1998;83: 1471-1482.

13 Masetti R, Biagi C, Zama D, Vendemini F, Martoni A, Morello W, Gasperini P, Pession A: Retinoids in pediatric onco-hematology: the model of acute promyelocytic leukemia and neuroblastoma. Adv Ther 2012;29:747-762.

14 Beckers D, Thomas M, Jamart J, Francois I, Maes M, Lebrethon MC, De Waele K, Tenoutasse S, De Schepper J: Adult final height after GH therapy for irradiation-induced GH deficiency in childhood survivors of brain tumors: the Belgian experience. Eur J Endocrinol 2010;162:483-490. 